\title{
Cellular schwannoma of the breast: A case report and review of literature
}

\author{
Yan Zhang* ${ }^{* 1}$, Hong-Yi Gao ${ }^{1}$, An-Qin Zhang ${ }^{2}$, Jiang-Yu Zhang ${ }^{1}$, Kun-He Wu ${ }^{1}$, Yi Wang ${ }^{1}$ \\ ${ }^{1}$ Department of Pathology, Guangdong Women and Children Hospital, Guangzhou, China \\ ${ }^{2}$ Breast Disease Center, Guangdong Women and Children Hospital, Guangzhou, China
}

Received: May 24, 2018

DOI: $10.5430 /$ crcp.v5n2p1
Accepted: July 1, 2018

URL: https://doi.org/10.5430/crcp.v5n2p1

Online Published: July 9, 2018

\begin{abstract}
Schwannoma of breast is a relatively uncommon disease characterized by painless and slow growing. We report a case of a 28-year-old female who present a mass of the right breast. Ultrasonography revealed a well-circumscribed and inhomogeneous hypoechoic mass under the skin of the breast. Then a biopsy and immunohistochemical analysis were performed. The results were suggestive for cellular schwannoma of breast. The tumour was removed successfully and the patient has been followed-up for six months with no evidence of recurrence. Cellular schwannoma is a rare benign neoplasm without metastasis. Distinction cellular schwannoma from other malignant tumours is very important. It is necessary to combine strict criteria on histological, immunohistochemical analysis, biological behavior, image examination and clinical features for the final diagnosis.
\end{abstract}

Key Words: Cellular schwannoma, Breast, Diagnosis

\section{INTRODUCTION}

Schwannoma is usually a benign tumour originating from schwann cells of the sheath of cranial, spinal nerve roots, peripheral and autonomic nerves. ${ }^{[1]}$ Schwannoma is commonly found in the flexor surface of the extremities, head, neck, retroperitoneum and mediastinum. ${ }^{[2-4]}$ Cellular schwannoma is discribed as an uncommon variant of schwannoma with hypercellularity, cytological atypia and presence of mitotic figures. ${ }^{[5]}$ It predominately affects middle-aged adults and develops mostly as a slow-growing tumour in the paravertebral region of the mediastinum and retroperitoneum. Schwannoma of breast is a rare tumour arising from the intercostal nerves. ${ }^{[6]}$ Breast cellular schwannoma has never been reported to date. Here, we first reported a rare case of cellular schwannomas of the breast, with review of literatures.

\section{Presentation of Case}

\subsection{Clinical presentation}

A 28-year-old Chinese female was found to have a mass in the right breast by ultrasonography examination four years ago. Now, she was recommended to our hospital for further examination. There were no skin findings, such as redness, orange peel-like change. Nipple retraction and nipple discharge were not observed. The mass was well-defined and mobile. No evidence of abnormal occurrence was present in axillary. The patient didn't have any features of von Recklinghausen's disease or family history of the disease. Ultrasonography revealed a well-circumscribed and inhomogeneous hypoechoic mass measuring $27 \mathrm{~mm} \times 58 \mathrm{~mm}$. The mass was at the 12-o'clock position of the right breast. It was under the skin and there was an obvious boundary to the mammary glands (see Figure 1).

\footnotetext{
*Correspondence: Yan Zhang; Email: peking105@163.com; Address: Department of Pathology, Guangdong Women and Children Hospital, 521 Xingnan Road, Guangzhou 511400, China. 

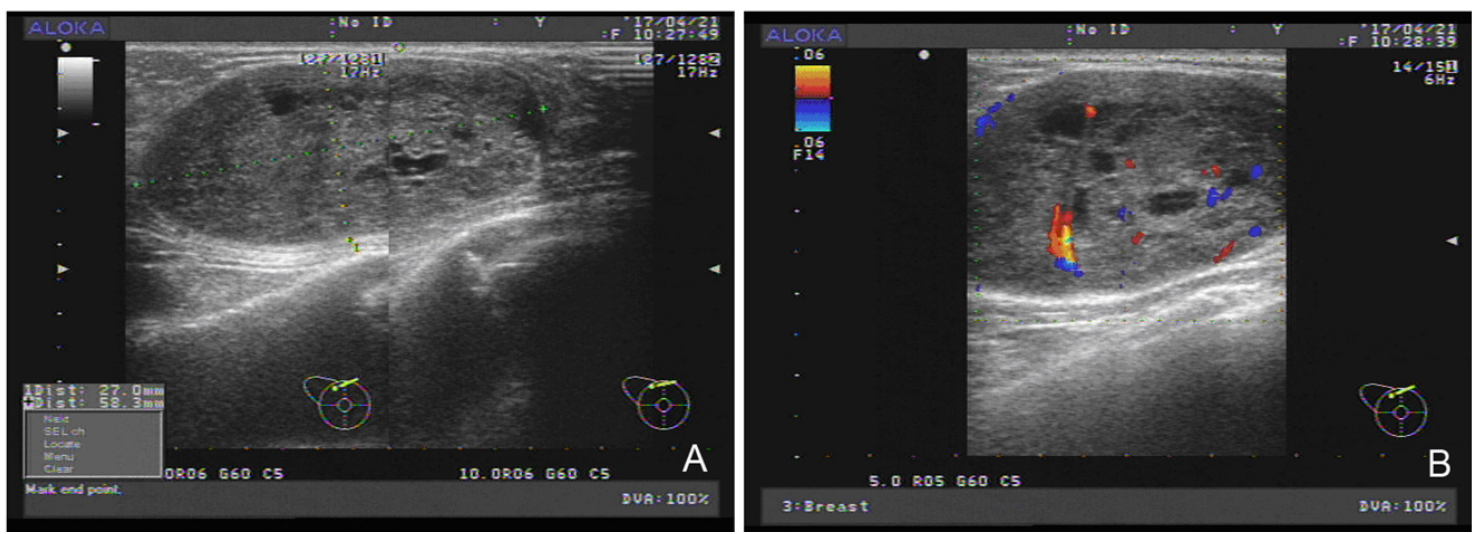

Figure 1. Ultrasonic image of the breast mass in this case. A. Ultrasonography revealed a well-circumscribed and inhomogeneous hypoechoic mass within the breast and adjacent to skin, measuring $27 \mathrm{~mm} \times 58 \mathrm{~mm}$. B. Colour doppler flow imaging is showing blood signals in the peripheral and inner region of the lesion

\subsection{Pathological findings}

A breast biopsy was performed under local anesthesia and specimens were sent to the laboratory for pathologic analysis. The tissue was fragment and envelope was not intact. Histopathological examination indicated predominantly hypercellular and few hypocellular areas. The hypercellular areas, known Antoni A tissue, were composed of a cellular spindle cell population with mild to moderate atypia. In this area the tumour cells had pointed basophilic nuclei with whorled arrangement. Population of atypical cells showed nuclear enlargement and overlap, coarse chromatin, nucleolar prominence. There was no necrosis and mitotic figures can be seen occasionally (see Figure 2A). Hypocellular areas, known as Antoni B tissue was scanty in this case. Small to large caliber hyalinized congested blood vessels were seen (see Figure 2B). Adjacent normal mammary glands was not visualized in the specimen. No evidence of invasion was present.

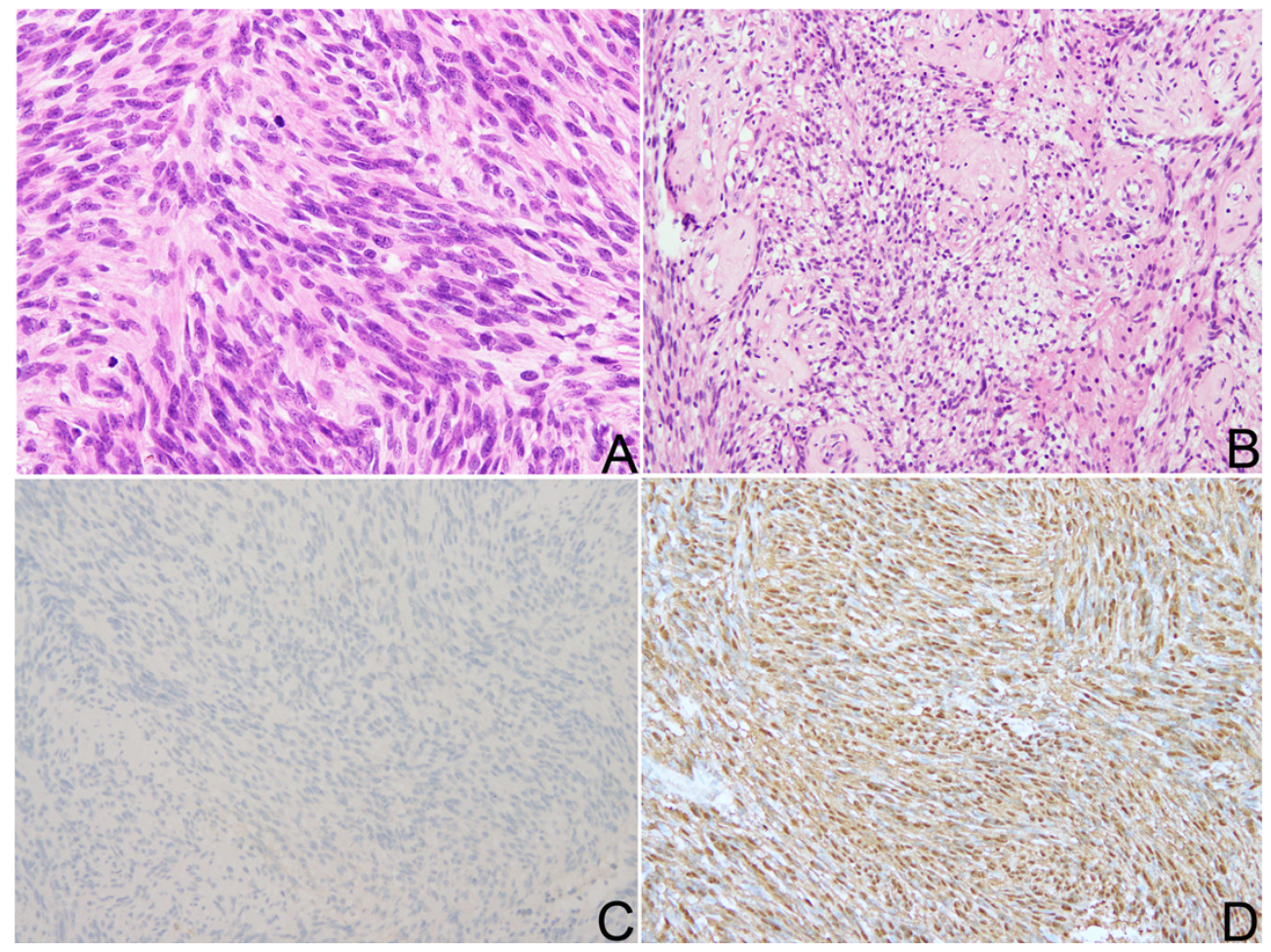

Figure 2. Hematoxylin-eosin and immunohistochemical staining of tissue from a patient with cellular schwannoma of the breast. Tumour cells showed whorled arrangement with nuclear enlargement, overlap and atypia, as shown in A, $\times 400$. The hyalinized congested blood vessels were shown in $\mathrm{B}, \times 200$. Immunohistochemical expression patterns of CK (C) and S-100 (D). The tumour was negative for CK and diffusely positive for S-100, $\times 200$. 
Immunohistochemical staining was strongly and diffusely positive for protein S-100 (see Figure 2D), Vimentin, Bcl-2 and $\beta$-catenin. The tumour was focally positive for NSE, CD68, weakly positive for p53, SMA and negative for CK (see Figure 2C), Desmin, CD34 and CD117. The Ki-67 index was $5 \%$. A final diagnosis of cellular schwannoma was made after clinical, histopathological and immunohistochemical correlation. During the follow-up period of 6 months, the patient remained well without recurrence.

\section{DisCuSSION}

A schwannoma is a relatively rare neoplasm derived from schwann cells of the nerve sheath. Schwannomas may occur throughout the body. The schwannomas of head and neck account for $25 \%-45 \%$ of all. ${ }^{[7]}$ Cellular schwannoma accounts for approximately $5 \%$ of the benign peripheral nerve sheath tumours. ${ }^{[5]}$ The tumours most commonly present at paravertebral sites, mediastinum, retroperitoneum and pelvis. However, a breast schwannoma is extremely rare. As a whole, there have been a few of reports about benign schwannoma of breast. ${ }^{[8-11]}$ Malignant schwannoma of the breast is unusual. ${ }^{[12-14]}$ However, the cellular schwannoma of breast have not been reported to date.

The clinical features of breast schwannoma were atypical. Usually the mass is asymptomatic, although tenderness or pain may occur in some instances. There were no skin changes or axillary lymphadenopathy. The preoperative diagnosis is often difficult and is made by ultrasonography, mammography, computed tomography (CT) and/or magnetic resonance imaging (MRI) to evaluate the size and the boundary of the tumour. Breast schwannoma appears as a well circumscribed mass, thereby mimicking a fibroadenoma or mesenchymal neoplasm. In our case, the clinical features and imaging description are similar to that of breast schwannoma reported previously. ${ }^{[8,9,15,16]}$

Microscopically, our findings from the excisional biopsy is different to conventional schwannoma by their prominent hypercellularity, lack of verocay bodies, nuclear hyperchromasia and a certain degree of atypical, a small number of mitotic figures $(<4 / 10 \mathrm{HPF}){ }^{[17]}$ The tumour cells were strongly and diffusely positive for protein S-100. It was suggestive of a cellular schwannoma.

Cellular schwannoma is an uncommon, benign peripheral nerve sheath tumour. Because of its hypercellularity, nuclear hyperchromasia and atypical, and the presence of mitotic figures, it is often misdiagnosed as malignant tumour. As a spindle cell lesion in the breast, the differential diagnosis of cellular schwannoma is a challenge. Spindle cell metaplastic carcinoma arises as first differential diagnosis in the present

Published by Sciedu Press case. In spindle cell metaplastic carcinoma, areas where the neoplastic cells form small clusters, with more epithelioid morphology or squamous differentiation can be found. In addition, the presence of ductal carcinoma in situ (DCIS) at the periphery or admixed with the lesion should prompt a diagnosis of metaplastic carcinoma. Since the histopathological characteristic of spindle cell metaplastic carcinoma is various. Sometime the presence of epithelial differentiation should be confirmed by immunohistochemical anlysis. p63 and keratin are positive in metaplastic carcinoma and negative in cellular schwannoma. In the case, specimen is sent fragmented to the pathology lab. The phyllodes tumour with minor epithelial components should be differentiated from cellular schwannoma. Phyllodes tumours is a group of generally circumscribed fibroepithelial neoplasms. Dignosis depends on finding residual epithelial structures. The tumour is positive for CD34, CD117, SMA and Desmin, while negative for S-100 in phyllodes tumour. Since cellular schwannoma is a monophasic lesion with pure spindle cells. Fibromatosis should also be differentiated. Fibromatosis, a benign lesion, is composed of an infiltrative growth of fibroblasts or myofibroblasts separated by collagen. Sometime focal lymphocytes is present and mitotic figures is rare.

In addition, another important differential diagnosis of cellular schwannoma is malignant peripheral nerve sheath tumour (MPNST). The presence of cellular whorls, perivascular and capsular lymphocytes and histocytes aggregates, and the absence of fascicles tend to the diagnosis of cellular schwannoma, while the presence of perivascular hypercellularity, extensive pleomorphism, high mitotic activity (> 10/10HPF), heterologous elements, and geographic areas of necrosis favor MPNST. ${ }^{[18]}$ Other malignant spindle cell tumour (dermatofibrosarcoma protuerans, leiomyosarcoma, fibrosarcoma, synovial sarcoma, malignant fibrous histiocytoma) should be differentiated from cellular schwannoma. These tumours often present malignant histologic features, such as significant polymorphism, high mitotic activity (> 10/HPF) and necrosis. Immunohistochemical staining for various markers is necessary. It is of great value in the differential diagnosis of these tumour.

In conclusion, cellular schwannoma is a rare benign neoplasm without metastasis. Currently, a complete excision of the tumour is an effective method of treatment and has a good prognosis. So distinction cellular schwannoma from other malignant tumours is very important. It is necessary to combine strict criteria on histological, immunohistochemical analyses, biological behavior, image examination and clinical features for the final diagnosis. 


\section{ACKNOWLEDGements}

This work was supported by Medical Scientific Research Foundation of Guangdong Province, China (Grant
No.A2016240).

\section{CONFlicts OF INTEREST Disclosure}

None.

\section{REFERENCES}

[1] Sharma S, Rai G. Schwannoma (Neurilemmoma) on the Base of the Tongue: A Rare Clinical Case. Am J Case Rep. 2016; 17: 203-6.

[2] Kim SH, Kim NH, Kim KR, et al. Schwannoma in head and neck: preoperative imaging study and intracapsular enucleation for functional nerve preservation. Yonsei Med J. 2010; 51(6): 938-42. https://doi.org/10.3349/ymj.2010.51.6.938201011938

[3] Wang W, Cui M, Ma HX, et al. A large schwannoma of the middle mediastinum: A case report and review of the literature. Oncol Lett. 2016; 11(3): 1719-21. https://doi.org/10.3892/ol.2016.41 48

[4] Lee EY, Kim JJ, Seok H, et al. Schwannoma of the tongue: a case report with review of literature. Maxillofac Plast Reconstr Surg. 2017; 39(1): 17. https://doi.org/10.1186/s40902-017-0116-2

[5] Woodruff JM, Godwin TA, Erlandson RA, et al. Cellular schwannoma: a variety of schwannoma sometimes mistaken for a malignant tumor. Am J Surg Pathol. 1981; 5(8): 733-44. PMid:7337161. https://doi.org/10.1097/00000478-198112000-00001

[6] Datta S, Pal A, Maiti M, et al. Rare case of chest wall schwannoma with destruction of rib, masquerading as a breast mass. J Clin Diagn Res. 2014; 8(6): FD01-2. https ://doi.org/10.7860/JCDR/201 4/6804.4416

[7] Suh JD, Ramakrishnan VR, Zhang PJ, et al. Diagnosis and endoscopic management of sinonasal schwannomas. ORL J Otorhinolaryngol Relat Spec. 2011; 73(6): 308-12. https://doi.org/10 $.1159 / 000331923$

[8] Halteh P, Patel A, Eskreis-Winkler S, et al. Schwannoma of the breast: A common tumor in an uncommon location. Breast J. 2017. https://doi.org/10.1111/tbj.12872

[9] Solano Diaz P, Hidalgo Martin MT, Sanchez Cordero MF, et al. Schwannoma of the breast: an unexpected diagnosis by magnetic resonance. Radiologia. 2017. https://doi.org/10.1016/j.rx .2017 .03 .004

[10] Parikh Y, Sharma KJ, Parikh SJ, et al. Intramammary schwannoma: a palpable breast mass. Radiol Case Rep. 2016; 11(3): 129-33. https : //doi.org/10.1016/j.radcr.2016.05.011

[11] Dialani V, Hines N, Wang Y, et al. Breast schwannoma. Case Rep Med. 2011; 2011: 930841. https://doi.org/10.1155/2011/9 30841

[12] Hauser H, Beham A, Steindorfer P, et al. Malignant schwannoma of the breast. Langenbecks Arch Chir. 1995; 380(6): 350-3. PMid:8559005.

[13] Berrada R, Chahtane A, Lakhdar A, et al. Malignant schwannoma of the breast. A case report. J Gynecol Obstet Biol Reprod (Paris). 1998; 27(4): 441-4.

[14] Besznyak I, Dubecz S, Peter I. Malignant schwannoma of the breast. Orv Hetil. 1998; 139(3): 137-9. PMid:9467297.

[15] Kalemoglu M, Demirbas S, Baloglu H, et al. Breast schwannoma. Breast J. 2006; 12(2): 184-5. https://doi.org/10.1111/j.10 75-122X.2006.00234.x

[16] Balci P, Pekcevik YT, Caferova S, et al. A case of benign schwannoma of the breast: mammographic, ultrasonographic and color Doppler ultrasonographic findings. Breast J. 2009; 15(4): 417-8. https://doi.org/10.1111/j.1524-4741.2009.00748.x

[17] Temime P, Tenenhaus, Privat Y, et al. Malignant Transformation of Recklinghausen's Royal Tumor of the Elbow (Malignant Schwannoma). Bull Soc Fr Dermatol Syphiligr. 1965; 72: 160-1. PMid:14335382.

[18] Alam K, Jain A, Misra A, et al. Cellular schwannoma masquerading as malignant peripheral nerve sheath tumour: a diagnostic dilemma. BMJ Case Rep. 2013; 2013. https : //doi .org/10.1136/bcr-2 012-008435 